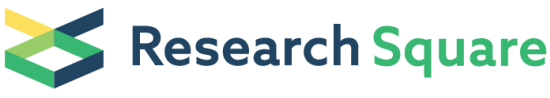 \\ Preprints are preliminary reports that have not undergone peer review. \\ They should not be considered conclusive, used to inform clinical practice, or referenced by the media as validated information.
}

\section{Comparison of the developmental potential of the morphological good embryos between normal- responders with low and high rate of MG day 3 embryos formation}

\section{Xiuliang Dai}

The affliated Changzhou Maternal and Child Health Care Hospital of Nanjing Medical Univeristy

\section{Xiyang Xia}

The affliated Changzhou Maternal and Child Health Care Hospital of Nanjing Medical University

\section{Tingting Gao}

The affiliated Changzhou Maternal and Child Health Care Hospital of Nanjing Medical University

\section{Chunmei Yu}

The affiliated Changzhou Maternal and Child Health Hospital of Nanjing Medical University

\section{Fang Cao}

The affliated Changzhou Maternal and Health Care Hospital of Nanjing Medical University

\section{Tianfu Li}

The affliated Changzhou Maternal and Health Care Hospital of Nanjing Medical University

\section{Lingjun Li}

The affiliated Changzhou Maternal and Child Health Care Hospital of Nanjing Medical University

\section{Yufeng Wang}

The affiliated Changzhou Maternal and Child Health Care Hospital of Nanjing Medical University

\section{Li Chen ( $\nabla$ czrcchenli@126.com )}

China Medical University Hospital

\section{Research}

Keywords: Embryonic quality, normal responders, rate of MG day 3 formation, day 3 embryos transfer, expanding culture

Posted Date: August 11th, 2021

DOl: https://doi.org/10.21203/rs.3.rs-794575/v1

License: @ (i) This work is licensed under a Creative Commons Attribution 4.0 International License. Read Full License 


\section{Abstract \\ Background}

To determine whether the morphological good (MG) day 3 embryos or blastocysts from normal responders with the low rate of MG day 3 embryos formation (LRTD3) and high rate of MG day 3 embryos formation (HRTD3) have similar developmental potential.

\section{Methods}

Oocyte retrieval cycles were ranked by the rate of MG day 3 embryos (MG day 3 embryos/2PN zygotes), cycles in the bottom25th percentile was defined as low potential group $(L)$ while cycles in the top 25th percentile was defined as high potential group $(H)$. The basic characteristics of patients and cycles, blastocyst formation rate, and clinical outcomes of transfer of the MG blastocysts or day 3 embryos were compared between the $\mathrm{H}$ and $\mathrm{L}$ groups.

\section{Results}

The overall characteristics of patients and cycles were grossly comparable between the $\mathrm{H}$ and $\mathrm{L}$ group in patients with MG day 3 embryo transfer or blastocysts transfer. In patients with transfer of MG day 3 embryos, patients with LRTD3 showed decreased rate of blastocyst formation and similar clinical outcomes of direct transfer of MG day 3 embryos, compared to patients with HRTD3. In patients with transfer of MG blastocysts, patients with LRTD3 showed decreased rate of blastocyst formation, as well as decreased rate of implantation and livebirth of transfer of MG blastocysts. compared to patients with HRTD3.

\section{Conclusion}

We concluded that the quality of MG day 3 embryos or MG blastocysts from patients with LRTD3 was reduced, compared to patients with HRTD3. However, it seems that direct transfer of MG day 3 embryos effectively avoid the flaws of poor quality of MG day 3 embryos from patients with LRTD3. Therefore, our study support the strategy of transfer of MG day 3 embryos rather than expended culture for patients with LRTD3.

\section{Introduction}

In vitro fertilization/embryo transfer (IVF/ET) technology is the most effective way for treatment of infertility. Many factors may influence the success of IVF, such as female age, duration of sub-fertility, bFSH and number of oocytes[1]. Among them, the number of oocytes is widely accepted as a maker indicating prognosis in the clinicians and patients. For a patient, more oocytes obtained may represent more available embryos for transfer, and more chance of having pregnancy within 1 oocyte retrieval cycle[2, 3].Previous studies have already showed that the live birth rate is strong associated with the number of oocytes[4].Ji et.al has demonstrated that for patient ( $=>16$ oocytes), although the fresh live birth rate per cycle is low, the cumulative live birth rate per cycle is the highest among groups[5]. Therefore the number of eggs takes the quantitative advantage. 
In clinical practice, the number of eggs is not always consistent with the number of available day 3 embryos, particularly the morphological good (MG) day 3embryos. For some patients, they may have a considerable number of oocytes but with poor in vitro development potential of fertilized oocytes, resulting in relatively few available MG day 3embryos. For this, the mood of patients may fall down to the bottom of the valley. It is known that quality may be as the same important as quantity in determining the success of IVF/ICSI cycles[6, 7]. It raises a question whether the MG embryos including the MG day 3 embryos and blastocysts from normal responders with low rate of MG day 3 embryos formation (LRTD3) and high rate of MG day 3 embryos formation (HRTD3) have similar embryonic quality. Having insight into this issue may help clinicians to develop more suitable and scientific strategy for the infertility treatment of normal responders with LRTD3 and to offer better consultations.

To address this issue, we respectively analyzed the data generated from Jan 2017 to May 2020 in our reproductive center. Oocyte retrieval cycles were ranked by the rate of MG day 3 embryos (MG day 3 embryos/ 2PN zygotes), cycles in the bottom25th percentile was defined as low potential group (L) while cycles in the top 25th percentile was defined as high potential group $(H)$. The basic characteristics of patients and cycles, blastocyst formation rate, and clinical outcomes of transfer of the MG blastocysts or day 3 embryos were compared between the $\mathrm{H}$ and $\mathrm{L}$ groups.

\section{Methods}

\section{Patients and study design}

A retrospective study was conducted in the reproductive medical center of the Nanjing Medical University Affiliated Changzhou Maternal and Child Health Care Hospital. Data generated from 908 patients who contributed a total of 916 fresh cycles and 1228 ET cycles (from Jan 2017 to May 2020) were included for final analysis. Actually, 1798 couples with 1851 fresh cycles were initially included for analysis. According to the rate of MG day 3 embryos (derived from 2 PN zygotes), cycles in the top 25th percentile were defined as the high group $(\mathrm{H})$ while cycles in the bottom 25th were defined as the low group $(\mathrm{L})$. Comparisons including the basic characteristics of patients and cycles, blastocyst formation, and clinical outcomes of transfer of MG blastocysts or day 3 embryos were made between the $\mathrm{H}$ and $\mathrm{L}$ groups.

\section{Inclusion/exclusion criteria}

1. Excluding the cycles with 2 PN-zygotes $<5$.

2. Excluding the cycles of PESA/TESA/late ICSI.

3. Male or female with a history of cancer or having chromosomal abnormality, or female having a diagnosis of recurrent spontaneous abortion.

4. Excluding cycles without expanding culture of day 3 embryos.

5. For ET cycles, only MG (MG) embryos including MG blastocysts or day 3 embryos were included for analysis. Morphologically non-good (MNG) embryos were excluded for analysis.

\section{Embryo culture procedures}

The ovarian stimulation protocols including long protocol, antagonist protocol, PPOS protocol and ministimulation protocol were conducted as previously described [8]. Thirty-six hours later, oocyte retrieval was 
performed after the trigger. Conventional IVF or ICSI method was used for insemination. Eighteen hours after insemination, zygotes with 2 pronuclei were defined as normal fertilization. The normal fertilization zygotes were transferred into G1 plus medium (Vitrolife, Sweden) for further culture. On day 3, the morphological score of the embryos was performed according to an established grading system[9]. In the present study, the MG day 3 embryos refer to Grade I and II embryos, while MNG day 3 embryos refer to Grade III embryos. Grade IV embryos was abandoned. MG or MNG day 3 embryos were either frozen by vitrification method, or for transfer, or for expanding culture. For expanding culture, day 3 embryos were transferred into drops of G2-plus medium (Vitrolife, Sweden). On day 5 or 6 , the embryos were undergone morphological score. Blastocyst with $C$ or less $C$ score of the inner cell mass and the trophectoderm was abandoned; blastocyst with A or B score of the inner cell mass and the trophectoderm was defined as the MG blastocyst; blastocyst with a $C$ score of either the inner cell mass or the trophectoderm and a A or B score of another (the trophectodermor the inner cell mass) was defined as the MNG blastocyst. The usable blastocysts represented the MG or MNG blastocysts. The usable blastocysts were frozen by vitrification or transferred into the uterine cavity. Furthermore, morphological evaluation of embryos in our reproductive center was performed by Dr. Yufeng Wang, who has more than ten years of work experience as an embryologist.

\section{Statistical analysis}

All analyses were performed using SPSS software (Version 21, IBM). For a comparison of the constituent ratio, the chi-square test was employed; for a comparison of the continuous data, the data were first examined by the Normality and Lognormality test. If data fitting the pattern of normally distributed, student $t$ test was employed, if not, the Mann-Whitney $U$ test was employed. For analysis of pregnancy and live birth, logistic regression analyses were used. Logistical regression analysis of clinical outcomes of day 3 cleavage embryos or blastocysts transfer, were adjusted for age of male or female, fresh or frozen ET cycles, number of embryos transferred, female BMI, sperm DFI to estimate the odds ratio(OR) with a $95 \% \mathrm{Cl}$ among the study groups.

\section{Results}

\section{The flow chart of the present study}

A total of 3905 fresh cycles/3090 couples from Jan 2017 to May 2020 in the reproductive medical center of Nanjing Medical University Affiliated Changzhou Maternal and Child Health Care Hospital was initially included in the present study (Fig.1). For the purpose of controlling the fluctuation of the rate of MG day 3 embryos (from 2PN), we excluded the cycles with $2 \mathrm{PN}$-zygotes $<5$. We further excluded the cycles of PESA/TESA/late ICSI. Male or female with a history of cancer or having chromosomal abnormality, or female having a diagnosis of recurrent spontaneous abortion was also excluded for analysis. Furthermore, data from blastocyst culture or transfer of blastocysts may provide important clues reflecting embryonic quality. Therefore, we excluded the cycles without blastocyst culture. After this, a total of 1851 fresh cycles/1798 couples was included for analysis (Fig.1). Then we rank the cycles according to the rate of MG day 3 embryos (from $2 \mathrm{PN}$ zygotes). The cycles in the bottom $25^{\text {th }}$ percentile were defined as the $\mathrm{L}$ group, while the cycles in the top $25^{\text {th }}$ percentile were defined as the $\mathrm{H}$ group. $\mathrm{A}$ total of 463 fresh cycles was included in the $L$ group while a total of 504 fresh cycles was included in the $\mathrm{H}$ group (Fig.1). In the $L$ group, 463 fresh cycles contributed 275 day 3 embryo transfer cycles and 282 blastocyst transfer cycles; in the $\mathrm{H}$ group, 504 fresh cycles contributed 189 day 3 embryo transfer cycles and 482 blastocyst transfer cycles (Fig.1). Given the uneven distribution of ET cycles containing MNG embryos between the $\mathrm{H}$ and $\mathrm{L}$ groups 
and a quite small fracture of ET cycles containing MNG embryos in the H group, therefore, we excluded the ET cycles containing MNG embryos for analysis. Finally, a total of 259 MG day 3 transfer cycles (from 242 fresh cycles)and 200 MG blastocyst transfer cycles (from 162 fresh cycles)was presented in the $L$ group, while a total of 189 MG day 3 transfer cycles (169 fresh cycles) and 426 MG blastocyst transfer cycles (from 343 fresh cycles) was presented in the $\mathrm{H}$ group (Fig.1). Data from these ET cycles were used for analysis of clinical outcomes of transfer of the MG blastocysts or day 3 embryos, while data from these fresh cycles were used for analysis of basic characteristics of couples, parameters of cycles and in vitro embryo development. As shown in Figure 2, 195vs.108 fresh cycles contributed only MG day 3 embryo transfer, 122 vs.289 fresh cycles contributed only MG blastocyst transfer and 47 vs.54 fresh cycles contributed both MG day 3 embryo and blastocyst transfer in the $\mathrm{L}$ vs. $\mathrm{H}$ group (Fig.2). Therefore, patients with transfer of MG day 3 embryos and patients with transfer of MG blastocyst represented relatively separate population included in the $\mathrm{L}$ and $\mathrm{H}$ groups. Therefore, parameters of couples, cycles and in vitro embryonic development were compared between the $\mathrm{L}$ and $\mathrm{H}$ groups in the patients with transfer of MG day 3 embryos or blastocysts separately.

\section{Parameters of couples, cycles and in vitro embryo development}

Baseline characteristics of couples or cycles were comparable between the $H$ and $L$ groups in patients with either MG day 3 transfer or blastocyst transfer, including the percentage of primary infertility, duration of infertility, primary diagnosis, age of males and females, the level of bFSH or AMH in females, AFC, BMI of males and females, semen DFI, the distribution of ovulation protocols, total dose of $G$ n and duration of Gn treatment (Table 1). E2 on the HCG day, oocytes retrieved, MII oocytes and 2-PN oocytes were lower in the H group than those in the $L$ group in patients with MG day 3 embryos transfer (Table 1). These parameters were similar in the $\mathrm{H}$ and $\mathrm{L}$ groups in the patients with transfer of blastocyst (Table 1). Viable day 3 embryos were significantly lower in the $\mathrm{H}$ group in patients with transfer of MG day 3 embryos, while viable day 3 embryos were significantly higher in the $\mathrm{H}$ group in patients with transfer of MG blastocysts (Table 1). MG day 3 embryos, rate of MG day 3 embryos, useable blastocysts and rate of useable blastocysts from MG day 3 embryos were significantly lower in the $L$ group than $\mathrm{H}$ group (Table 1).

\section{Characteristics of MG blastocyst transfer and clinical outcomes}

More number of MG blastocysts per ET cycle and decreased rate of ET cycles with single blastocyst were observed in the $\mathrm{H}$ group (Table 2). However,the rate of clinical pregnancy, live birth, implantation and live birth per embryo was significantly lower in the $\mathrm{L}$ group than in the $\mathrm{H}$ group (Table 2 ). Abortion rate was similar between the two groups (Table 2). To adjust the confounding factors that potentially affect the clinical pregnancy outcomes (including female or male age, fresh or frozen ET cycles, number of embryos transferred, female BMI, semen DFI), we employed the logistic regression analysis. Our results showed that the origination of MG blastocyst (from the $\mathrm{L}$ group or the $\mathrm{H}$ group) were associated the clinical pregnancy outcomes (Table 2).

\section{Characteristics of MG day3 embryo transfer and clinical outcomes}

Although more embryos per ET cycle was observed in the $\mathrm{H}$ group, the rate of singleton, pregnancy, abortion, live birth, implantation and live birth per embryo was comparable between the $\mathrm{H}$ and $\mathrm{L}$ groups (Table 3 ). The logistic regression analysis was used to adjust the confounding factors, including female or male age, fresh or frozen ET cycles, number of embryos transferred, female BMI, semen DFI. Our results showed that the origination of MG day 3embryos (from the L group or the H group) had no effect on the clinical pregnancy outcomes (Table 3). 


\section{Summary of the main findings}

Our results showed that in patients with transfer of MG day 3 embryos, patients with LRTD3 showed decreased rate of blastocyst formation and similar clinical outcomes of direct transfer of MG day3 embryos, compared to patients with HRTD3 (Figure 3A). In patients with transfer of MG blastocysts, patients with LRTD3 showed decreased rate of blastocyst formation, as well as decreased rate of implantation and livebirth of transfer of MG blastocysts (Figure 3B).

\section{Discussion}

In the present study, we found that the usable blastocyst formation rate (derived from the MG day 3 embryos), as well as the in vivo developmental potential of MG blastocysts was reduced significantly in the $L$ group, compared to the $\mathrm{H}$ group. However, transfer of the MG day 3 embryos resulted in comparable clinical outcomes in the $\mathrm{H}$ and L groups. Our study support the strategy of transfer of MG day 3 embryos instead of extended culture for treatment of patients with LRTD3, particularly when few MG embryos were available on day 3.

A previous study showed that the number of good quality embryos available on day 3 over 3 is a strong predictive value for both pregnancy rate and implantation rate [10]. Different from that study, we grouped the patients/cycles by rate of MG embryos on day 3 in the present study. This is a new perspective focusing on the capacity of overall MG embryos formation on day 3 rather than the absolute counts of MG day 3 embryos. The follicles within a patient promoted by controlled ovarian hyperstimulation share a common growth environment and genetic foundation. Therefore, we assumed that rate of MG day 3 embryos may better reflect the quality of follicles, rather than the absolute counts. To clarify this hypothesis, the developmental potential of MG embryos from patients with low and high RTD3 was compared. For controlling the fluctuation of rate of MG day 3 embryos, patients underwent a fresh cycle with 2 PN-zygotes $=>5$ were included for analysis. Therefore patients included in this study were normal responders with relatively good prognosis.

In the present, we found that fresh cycles with transfer of MG day 3 embryos is distinct from fresh cycles with blastocyst transfer, and only a small fracture of fresh cycles have both transfer of MG day3 embryos and blastocysts in either the $\mathrm{L}$ or $\mathrm{H}$ groups. This was due to the transfer strategy. In our reproductive center, 2-4 MG day 3 embryos (if available) will be frozen or transferred, and the remaining will be further cultured. For embryo selection, MG blastocyst has the highest priority, followed by MG day 3 embryos, the next is MNG blastocyst, and the last is MNG day 3 embryos. Patients having frozen MG day 3 embryo transfer may indicate no available MG blastocysts or no extra MG embryos for further culture. The baseline characteristics of patients and consequent ovulation protocols were comparable between the $L$ and $H$ groups in patients with MG blastocyst and day 3 embryo transfer. However, we noticed that patients with transfer of MG blastocysts have higher ovarian reserve and ovarian response, compared to patients with transfer of MG day 3 embryos. This is reasonable. Because with similar RTD3, more number of eggs means more chance having MG blastocysts.

For patients with day 3 embryos transfer, although similar $\mathrm{Gn}$ use, the number of mature follicles and oocyte retrieved were significantly lower in the $\mathrm{H}$ group than that in the $\mathrm{L}$ group, indicating relatively low response of patients in the $\mathrm{H}$ group, compared to patients in the $\mathrm{L}$ group. However, the reduced oocytes retrieved in the $\mathrm{H}$ group were compensated by the HRTD3, resulting in more available MG embryos on day 3 and subsequent more MG day 3 embryos for expended culture. For patients with MG blastocyst transfer, the only difference between 
the two groups was the RTD3. HRTD3 resulted in more available MG embryos on day 3 and more MG day 3 embryos for expended culture in the $\mathrm{H}$ group. Taken all together, grouping by RTD3 didn't introduce the obvious confounding factors regarding baseline characteristics of patients and cycles.

Usable blastocyst formation rate is a clinically important parameter reflecting the developmental potential of embryos. In the present study, we observed a higher of usable blastocyst formation rate in patients with transfer of blastocyst than in patients with transfer of MG day3 embryos. It has been reported that more dose of $\mathrm{Gn}$ use adversely affect the quality of eggs[11, 12]. Therefore, it is possible that high dose of $\mathrm{Gn}$ use in patients with MG day 3 embryo transfer deteriorates the oocyte quality. However, we found that usable blastocyst formation rate from MG day 3 embryos was reduced significantly in the $L$ group, compared to the $H$ group in either the MG day 3 embryo transfer or blastocyst transfer group, indicating that MG day 3 embryos from patients with low RTD3 showed decreased ability of being usable blastocyst in vitro, compared to patients with HRTD3.

The golden maker reflecting the quality of embryo is whether the embryo can result in a live birth [13]. Although a decreased ability of being usable blastocyst by in vitro culture of the MG day 3 embryos from the patients with LRTD3 was observed, how about the in vivo developmental potential of MG blastocysts from the patients with LRTD3? In the present study, we found that not only clinical pregnancy rate and livebirth rate, but also implantation rate and livebirth rate per embryo were significantly higher in the $\mathrm{H}$ group than the $\mathrm{L}$ group. It is know that female age and BMI, number of embryos transferred and semen DFI have profound influences on the clinical outcomes of in vitro fertilization [13-19]. In addition, we found that there was a trend towards decreased male age in the $\mathrm{H}$ group, compared with $\mathrm{L}$ group. Although conflicting results were reported regarding the effect of male age on the clinical outcomes of in vitro fertilization, the factor of male age should be also considered [20, 21]. After adjusted for age of male or female, number of embryos transferred, female BMI and sperm DFI, we found that the embryos from the $\mathrm{H}$ group was positively associated with the clinical pregnancy and livebirth. Therefore, although similar morphological appearance (they were both MG blastocyst), the MG blastocysts from patients with LRTD3 showed reduced in vivo developmental potential, compared to that from patients with HRTD3.

To our surprise, transfer of MG day 3 embryos resulted in similar clinical outcomes in the $H$ and $L$ groups. After adjusted for factors including male or female age, number of embryos transferred, female BMI and sperm DFI, type of embryo transfer (fresh or frozen transfer), we found that embryos from the $\mathrm{H}$ group were not associated with the clinical pregnancy and livebirth. This was quite different from the results from blastocyst transfer. In addition, this result was also paradox with the finding that a decreased useful blastocyst formation rate was observed in the $L$ group for group of $M G$ day 3 embryo transfer. One reasonable explanation is the difference of developmental potential of MG day 3 embryos resulted from in vitro and in vivo. It has been proposed that the day 3 embryos which can't develop into blastocysts in vitro may lead to live births in vivo [22]. Therefore, we deduced that that MG day 3 embryos from patients with LRTD3 is more sensitive to in vitro culture but direct transfer, resulting in decreased useable blastocyst formation rate and similar clinical outcomes of direct transfer of MG day 3 embryos, compared with patients with high LRTD3.

Data from direct MG day 3 embryo transfer showed that patients with LRTD3 had limited MG day 3 embryos with a decreased rate of useful blastocyst formation rate compared to patients with HRTD3. However, direct transfer of MG day 3 embryos from patients with LRTD3 may achieve similar clinical pregnancy outcomes as patients with HRTD3. Data from transfer of blastocyst showed that rate of blastocyst formation is significantly reduced in 
patients with LRTD3. More importantly, transfer of MG blastocyst resulted in poorer clinical pregnancy outcomes in patients with LRTD3 than patients with HRTD3. Therefore, we concluded that the quality of MG day 3 embryos or blastocysts from patients with LRTD3 was reduced compared to patients with HRTD3. However, it seems that direct transfer of MG day 3 embryos effectively avoid the flaw of poor quality of MG day 3 embryos from patients with RTD3.

The main limitation of this study is its nature of the retrospective study. We noticed that transfer of MG day 3 embryos and transfer of blastocysts represented relatively separate population in either the $L$ group or the $\mathrm{H}$ group. Population of transfer of blastocyst had higher response to $\mathrm{Gn}$ and less $\mathrm{Gn}$ use, more oocytes retrieved and higher blastocyst formation rate, compared to patients with transfer of MG day 3 embryos. A concern is that whether it is appropriate that results from separate populations were integrated for analysis. This study was a comparison study, did not provide the direct evidence. Furthermore, the data included for analysis were from single center and the cases included in the present study is limited, therefore, the indications from this study need to be confirmed by randomized control trial.

\section{Conclusion}

Here, we reported that a decreased ability of being useful blastocysts were observed in patients with LRTD3 in group of either transfer of MG day 3 embryos or in group of transfer of blastocysts, compared to patients with HRTD3. A decreased in vivo developmental potential of MG blastocysts and a similar in vivo developmental potential of MG day 3 embryos were observed in patients with LRTD3, compared to patients with HRTD3. Therefore, we concluded that the quality of MG day 3 embryos or blastocysts from patients with LRTD3 was reduced compared to patients with HRTD3. However, it seems that direct transfer of MG day 3 embryos effectively avoid the flaws of poor quality of MG day 3 embryos from patients with RTD3. Therefore, our study support the strategy of transfer of MG day 3 embryos instead of expended culture for patients with low RTD3.

\section{Abbreviations}

LRTD3: Low rate of morphological good embryos formation; HRTD3: High rate of morphological good embryos formation; MG: Morphologically good ; MNG: Morphologically non-good

\section{Declarations}

\section{Author's contributions}

Substantial contribution to conception and design: L.C., X.D. and Y.W. ; Data acquisition: X.X., T.G. F.C; Data analysis: X.X., T.G.,C.Y.,F.C., T.L. and L.L; Data interpretation: all authors. Drafting the article: X.D. and Y.W. ;Critical revision of the article for important intellectual content: all authors. Final approval of the article: all authors.

\section{Funding}

This study was supported by the National Natural Science Foundation of China (No.81901436, to X.D), Changzhou Health Committee Funded Young Investigator Training Project (CZQM2020094 to X.D.\& CZQM2020099 to T.G.), Key Program of Changzhou Municipal Health Commission (ZD201921 to L.C.), Program 
of Jiangsu Province's Key Provincial Talents of Women and Child Health Care (FRC 201751 to L.C.) and Jiangsu Population Institute funded program (JSPA2019017 to T.L.).

\section{Availability of data and materials}

The datasets used and/or analysed during the current study are available from the corresponding author on reasonable request.

\section{Ethics approval and consent to participate}

All of the included patients read and signed the informed consent form. This retrospective study was approved by the Ethics Committee of Changzhou Maternal and Child Health Care Hospital and Nanjing Medical University. All the treatments in the present study were performed strictly in accordance with the Declaration of Helsinki for Medical Research.

\section{Consent for publication}

All participants gave written consent to use of their data for research purposes and publication.

\section{Competing interests}

The authors have no competing interests

\section{Acknowledgments}

Not applicable

\section{References}

1. Van Loendersloot LL, Van Wely M, Limpens J, Bossuyt PM, Repping S, Van Der Veen F. Predictive factors in in vitro fertilization (IVF): a systematic review and meta-analysis. Hum Reprod Update. 2010;16(6):577-89.

2. Drakopoulos P, Errazuriz J, Santos-Ribeiro S, Tournaye H, Vaiarelli A, Pluchino N, et al. Cumulative live birth rates in in-vitro fertilization. Minerva Ginecol. 2019;71(3):207-10.

3. Polyzos NP, Drakopoulos P, Parra J, Pellicer A, Santos-Ribeiro S, Tournaye H, et al. Cumulative live birth rates according to the number of oocytes retrieved after the first ovarian stimulation for in vitro fertilization/intracytoplasmic sperm injection: a multicenter multinational analysis including approximately 15,000 women. Fertil Steril. 2018;110(4):661-70 e1.

4. Sunkara SK, Rittenberg V, Raine-Fenning N, Bhattacharya S, Zamora J, Coomarasamy A. Association between the number of eggs and live birth in IVF treatment: an analysis of 400135 treatment cycles. Hum Reprod. 2011;26(7):1768-74.

5. Ji J, Liu Y, Tong XH, Luo L, Ma J, Chen Z. The optimum number of oocytes in IVF treatment: an analysis of 2455 cycles in China. Hum Reprod. 2013;28(10):2728-34.

6. Oron G, Son WY, Buckett W, Tulandi T, Holzer H. The association between embryo quality and perinatal outcome of singletons born after single embryo transfers: a pilot study. Hum Reprod. 2014;29(7):1444-51. 
7. Li J, Chen Q, Wang J, Huang G, Ye H. Does growth hormone supplementation improve oocyte competence and IVF outcomes in patients with poor embryonic development? A randomized controlled trial. BMC Pregnancy Childbirth. 2020;20(1):310.

8. Dai X, Wang Y, Yang H, Gao T, Yu C, Cao F, et al. AMH has no role in predicting oocyte quality in women with advanced age undergoing IVF/ICSI cycles. Sci Rep. 2020;10(1):19750.

9. Bungum M, Bungum L, Humaidan P. A prospective study, using sibling oocytes, examining the effect of 30 seconds versus 90 minutes gamete co-incubation in IVF. Hum Reprod. 2006;21(2):518-23.

10. Volpes A, Sammartano F, Coffaro F, Mistretta V, Scaglione P, Allegra A. Number of good quality embryos on day 3 is predictive for both pregnancy and implantation rates in in vitro fertilization/intracytoplasmic sperm injection cycles. Fertil Steril. 2004;82(5):1330-6.

11. Santos MA, Kuijk EW, Macklon NS. The impact of ovarian stimulation for IVF on the developing embryo. Reproduction. 2010;139(1):23-34.

12. Roberts R, latropoulou A, Ciantar D, Stark J, Becker DL, Franks S, et al. Follicle-stimulating hormone affects metaphase I chromosome alignment and increases aneuploidy in mouse oocytes matured in vitro. Biol Reprod. 2005;72(1):107-18.

13. Ata B, Seyhan A, Seli E. Diminished ovarian reserve versus ovarian aging: overlaps and differences. Curr Opin Obstet Gynecol. 2019;31(3):139-47.

14. Zhang JJ, Liu X, Chen L, Zhang S, Zhang X, Hao C, et al. Advanced maternal age alters expression of maternal effect genes that are essential for human oocyte quality. Aging. 2020;12(4):3950-61.

15. Sermondade N, Huberlant S, Bourhis-Lefebvre V, Arbo E, Gallot V, Colombani M, et al. Female obesity is negatively associated with live birth rate following IVF: a systematic review and meta-analysis. Hum Reprod Update. 2019;25(4):439-51.

16. Kasum M, Oreskovic S, Cehic E, Lila A, Ejubovic E, Soldo D. The role of female obesity on in vitro fertilization outcomes. Gynecol Endocrinol. 2018;34(3):184-8.

17. Pandian Z, Marjoribanks J, Ozturk O, Serour G, Bhattacharya S. Number of embryos for transfer following in vitro fertilisation or intra-cytoplasmic sperm injection. Cochrane Database Syst Rev. 2013;7:CD003416.

18. Oleszczuk K, Giwercman A, Bungum M. Sperm chromatin structure assay in prediction of in vitro fertilization outcome. Andrology. 2016;4(2):290-6.

19. Micinski P, Pawlicki K, Wielgus E, Bochenek M, Tworkowska I. The sperm chromatin structure assay (SCSA) as prognostic factor in IVF/ICSI program. Reprod Biol. 2009;9(1):65-70.

20. Mariappen U, Keane KN, Hinchliffe PM, Dhaliwal SS, Yovich JL. Neither male age nor semen parameters influence clinical pregnancy or live birth outcomes from IVF. Reprod Biol. 2018;18(4):324-9.

21. Van Opstal J, Fieuws S, Spiessens C, Soubry A. Male age interferes with embryo growth in IVF treatment. Hum Reprod. 2021;36(1):107-15.

22. Gleicher N, Kushnir VA, Barad DH. Is it time for a paradigm shift in understanding embryo selection? Reprod Biol Endocrinol. 2015;13(3).

\section{Tables}

Table 1 Baseline characteristics of couples, parameters of cycles and in vitro 


\section{embryo development}

Data are presented as the median [the first quartile, the third quartile] or count (percentage).

DFI DNA fragmentation index; Gn: gonadotropin; AFC antral follicle count; MG: morphologically good *Semen was analyzed by CASA 2 weeks before oocyte retrieval.

Table 2 Parameters of MG blastocyst transfer and the association between the groups and clinical outcomes

\begin{tabular}{|c|c|c|c|}
\hline & $\mathbf{L}$ & $\mathrm{H}$ & $\mathbf{P}$ \\
\hline ET cycles & 200 & 426 & \\
\hline Embryos /transfer & $1.27 \pm 0.45$ & $1.47 \pm 0.50$ & $<0.0001$ \\
\hline Single embryo transfer cycles (\%) & $146(73.00)$ & $225(52.82)$ & $<0.0001$ \\
\hline Pregnancy rate (\%) & $118(59.00)$ & $318(74.65)$ & $<0.0001$ \\
\hline Abortion rate (\%) & $19(16.10)$ & $47(14.78)$ & 0.7322 \\
\hline Live birth rate (\%) & $98(49.00)$ & $271(63.62)$ & $<0.0001$ \\
\hline Implantation rate (\%) & $137 / 254(53.94)$ & $406 / 627(64.75)$ & 0.0028 \\
\hline Live birth per embryo (\%) & $114 / 254(44.88)$ & $341 / 627(54.39)$ & 0.0106 \\
\hline Clinical pregnancy & 1 (Ref) & $1.907(1.305-2.787)$ & .001 \\
\hline Live birth\# & 1 (Ref) & $1.676(1.169-2.404)$ & .005 \\
\hline Abortion\# & 1 (Ref) & $0.926(0.508-1.688)$ & .314 \\
\hline
\end{tabular}

Data are presented as the median [the first quartile, the third quartile] or count (percentage) or Mean \pm SD or odds ratio $(\mathrm{OR})(95 \% \mathrm{Cl})$.

\# Logistic regression models adjusted for age of male or female, fresh or frozen ET cycles, number of embryos transferred, female BMI, sperm DFI to estimate the OR of pregnancy outcomes, including clinical pregnancy, live birth and abortion.

ET Embryo transfer; MG: morphologically good.

Blastocyst with A or B score of the inner cell mass and the trophectoderm was defined as the MG blastocyst.

Table 3 Parameters of MG day 3 embryo transfer and the association between the groups and clinical outcomes 
MG day 3 embryo transfer

\begin{tabular}{lllllll} 
& $\mathrm{L}$ & $\mathrm{H}$ & $\mathrm{p}$ & $\mathrm{L}$ & $\mathrm{H}$ & $\mathrm{p}$ \\
\hline Cycles(n) & 242 & 162 & $/$ & 169 & 343 & $/$ \\
\hline Couples (n) & 235 & 162 & $/$ & 168 & 343 & $/$ \\
\hline $\begin{array}{l}\text { Primary } \\
\text { infertility (\%) }\end{array}$ & $137(56.61)$ & $89(54.94)$ & 0.7399 & $92(54.44)$ & $193 / 343(56.27$ & 0.695 \\
\hline $\begin{array}{l}\text { Infertility } \\
\text { duration } \\
\text { (years) }\end{array}$ & $3[2,5]$ & $3[2,5]$ & 0.3449 & $3[2,5]$ & $3[2,4]$ & 0.2913 \\
\hline
\end{tabular}

Age (years)

\begin{tabular}{lllllll} 
Female & $30[28,33]$ & $30[28,33.25]$ & 0.4795 & $30[28,33]$ & $30[28,33]$ & 0.5762 \\
Male & $31[29,35]$ & $30[28,35]$ & 0.0556 & $32[29,35]$ & $31[29,34]$ & 0.0602 \\
\hline BMI(kg/m2) & & & & & & \\
\hline Female & $22.10[20.20$, & $22.4[20.5$, & 0.3971 & $21.85[19.9,24.3]$ & $22[20,24.9]$ & 0.3937 \\
& $24.75]$ & $25.2]$ & & & & \\
Male & 24.4 & 24.7 & 0.9434 & 24.2 & $24.55[22,27.18]$ & 0.2003 \\
& {$[22.3,27.4]$} & {$[22.4,27.23]$} & & {$[21.53,26.78]$} & & \\
\hline Semen DFI & 10.61 & 10.30 & 0.5187 & $9.97[6.16,15.11]$ & $10.41[6.7,16.4]$ & 0.2357 \\
& {$[7.213,16.46]$} & {$[6.848,14.99]$} & & & & \\
\hline AFC & $10[7,14]$ & $10[8,14]$ & 0.8166 & $11[8,14]$ & $11.5[8,16]$ & 0.6474
\end{tabular}

Primary

diagnosis

\begin{tabular}{lllllll}
\hline Tubal & $110(45.45)$ & $84(51.85)$ & 0.2072 & $84(49.70)$ & $185(53.94)$ & 0.3672 \\
$\begin{array}{l}\text { Ovulation } \\
\text { defect }\end{array}$ & $28(11.57)$ & $23(14.20)$ & 0.4358 & $25(14.79)$ & $49(14.29)$ & 0.878 \\
\hline $\begin{array}{l}\text { Male factor } \\
\text { others }\end{array}$ & $40(16.53)$ & $17(10.49)$ & 0.0877 & $20(11.83)$ & $39(11.37)$ & 0.8771 \\
\hline $\begin{array}{l}\text { Female } \\
\text { hormonal } \\
\text { level }\end{array}$ & $64(26.45)$ & $38(23.46)$ & 0.4979 & $40(23.67)$ & $70(20.41)$ & 0.3983 \\
\hline $\begin{array}{l}\text { Basal FSH, } \\
\text { IU/l }\end{array}$ & 6.43 & 6.615 & 0.5462 & $5.96[5.13,7.12]$ & $6.17[5.263,7.452]$ & 0.1582 \\
\hline $\begin{array}{l}\text { AMH, ng/ml } \\
{[5.61,7.50]}\end{array}$ & {$[5.563,7.88]$} & & & & \\
\hline $\begin{array}{l}\text { GnRH } \\
\text { analogues(\%) }\end{array}$ & {$[2.203,5.568]$} & {$[1.968,5.363]$} & 0.5555 & $4.07[2.765,6.5]$ & $4.43[1.848,6.908]$ & 0.5056 \\
\hline Agonist & $177(73.14)$ & $121(74.69)$ & 0.7284 & $140(84.02)$ & $280(81.63)$ & 0.5039 \\
\hline Antagonist & $40(16.53)$ & $28(17.28)$ & 0.8424 & $27(15.98)$ & $60(17.49)$ & 0.6675 \\
\hline
\end{tabular}




\begin{tabular}{|c|c|c|c|c|c|c|}
\hline Others & $25(10.33)$ & $13(8.03)$ & 0.095 & $0(0)$ & $3(0.87)$ & 0.2227 \\
\hline $\begin{array}{l}\text { Total dose of } \\
\text { Gn (IU) }\end{array}$ & $\begin{array}{l}1706 \\
{[1263,2400]}\end{array}$ & $\begin{array}{l}1931 \\
{[1322,2400]}\end{array}$ & 0.3312 & $\begin{array}{l}1500 \\
{[1075,2100]}\end{array}$ & $\begin{array}{l}1425 \\
{[1100,2138]}\end{array}$ & 0.8374 \\
\hline $\begin{array}{l}\text { Duration of } \\
\text { Gn treatment } \\
\text { (days) }\end{array}$ & $9[8,10]$ & $9[8,10]$ & 0.5967 & $9[8,10]$ & $9[8,10]$ & 0.7377 \\
\hline $\begin{array}{l}\text { E2 on the } \\
\text { HCG day, ng/l }\end{array}$ & $\begin{array}{l}3009 \\
{[2222,4244]}\end{array}$ & $\begin{array}{l}2695 \\
{[1878,3912]}\end{array}$ & 0.0157 & $\begin{array}{l}3705 \\
{[2606,5233]}\end{array}$ & $\begin{array}{l}4007 \\
{[2640,5282]}\end{array}$ & 0.7573 \\
\hline $\begin{array}{l}\text { Oocytes } \\
\text { retrieved }\end{array}$ & $11[9,13]$ & $9[7,11]$ & $<0.0001$ & $12[10,16]$ & $13[10,15]$ & 0.616 \\
\hline MIl oocytes & $10[8,12]$ & $9[7,11]$ & $<0.0001$ & $12[9,15]$ & $12[9,14]$ & 0.7644 \\
\hline 2PN zygotes & $8[6,10]$ & $7[6,9]$ & 0.0002 & $10[7,12]$ & $10[7,12]$ & 0.7305 \\
\hline $\begin{array}{l}\text { Viable day } 3 \\
\text { embryos }\end{array}$ & $7[5,8]$ & $7[6,9]$ & 0.0471 & $8[6,10]$ & $10[7,11]$ & $<0.0001$ \\
\hline $\begin{array}{l}\text { MG day } 3 \\
\text { embryos }\end{array}$ & $3[2,4]$ & $7[6,9]$ & $<0.0001$ & $4[3,6]$ & $9[7,11]$ & $<0.0001$ \\
\hline $\begin{array}{l}\text { MG day } 3 \\
\text { embryos } \\
\text { from 2PN (\%) }\end{array}$ & $\begin{array}{l}836 / 2065 \\
(40.48)\end{array}$ & $\begin{array}{l}1183 / 1214 \\
(97.45)\end{array}$ & $<0.0001$ & $\begin{array}{l}825 / 1705 \\
(48.39)\end{array}$ & $\begin{array}{l}3266 / 3418 \\
(95.55)\end{array}$ & $<0.0001$ \\
\hline $\begin{array}{l}\text { Usable } \\
\text { blastocysts }\end{array}$ & $1[0,2]$ & $3[2,4]$ & $<0.0001$ & $3[2,4]$ & $5[4,7]$ & $<0.0001$ \\
\hline $\begin{array}{l}\text { Usable } \\
\text { blastocysts } \\
\text { formation } \\
\text { MG day } 3 \\
\text { embryos (\%) }\end{array}$ & $\begin{array}{l}171 / 321 \\
(53.27)\end{array}$ & $\begin{array}{l}476 / 769 \\
(61.90)\end{array}$ & 0.0082 & $\begin{array}{l}401 / 585 \\
(68.58)\end{array}$ & $\begin{array}{l}1910 / 2630 \\
(72.62)\end{array}$ & 0.0473 \\
\hline
\end{tabular}

\begin{tabular}{|llll|}
\hline & L & H & P \\
\hline ET cycles & 259 & 189 & \\
\hline Number of embryos per transfer & $1.88 \pm 0.32$ & $1.98 \pm 0.14$ & 0.0002 \\
\hline Pregnant rate (\%) & $145(55.98)$ & $111(58.73)$ & 0.562 \\
\hline Abortions rate (\%) & $21(14.48)$ & $14(12.61)$ & 0.666 \\
\hline Live birth rate (\%) & $124(47.88)$ & $97(51.32)$ & 0.4712 \\
\hline Implantation rate (\%) & $193 / 488(39.55)$ & $157 / 374(41.98)$ & 0.4716 \\
\hline Live birth per embryo (\%) & $159 / 488(32.58)$ & $133 / 374(35.56)$ & 0.3596 \\
\hline Clinical pregnancy $\#$ & 1 (Ref) & $1.054(0.707-1.572)$ & 0.796 \\
\hline Live birth\# & 1 (Ref) & $1.093(0.736-1.625)$ & 0.659 \\
\hline Abortion $^{\#}$ & 1 (Ref) & $0.846(0.397-1.801)$ & 0.664 \\
\hline
\end{tabular}


Data are presented as the median [the first quartile, the third quartile] or count (percentage) or Mean \pm SD or odds ratio $(95 \% \mathrm{Cl})$.

\#Logistic regression models adjusted for age of male or female, fresh or frozen ET cycles, number of embryos transferred, female BMI, sperm DFI to estimate the OR of pregnancy outcomes, including clinical pregnancy, live birth and abortion.

ET Embryo transfer; MG: morphologically good

\section{Figures}

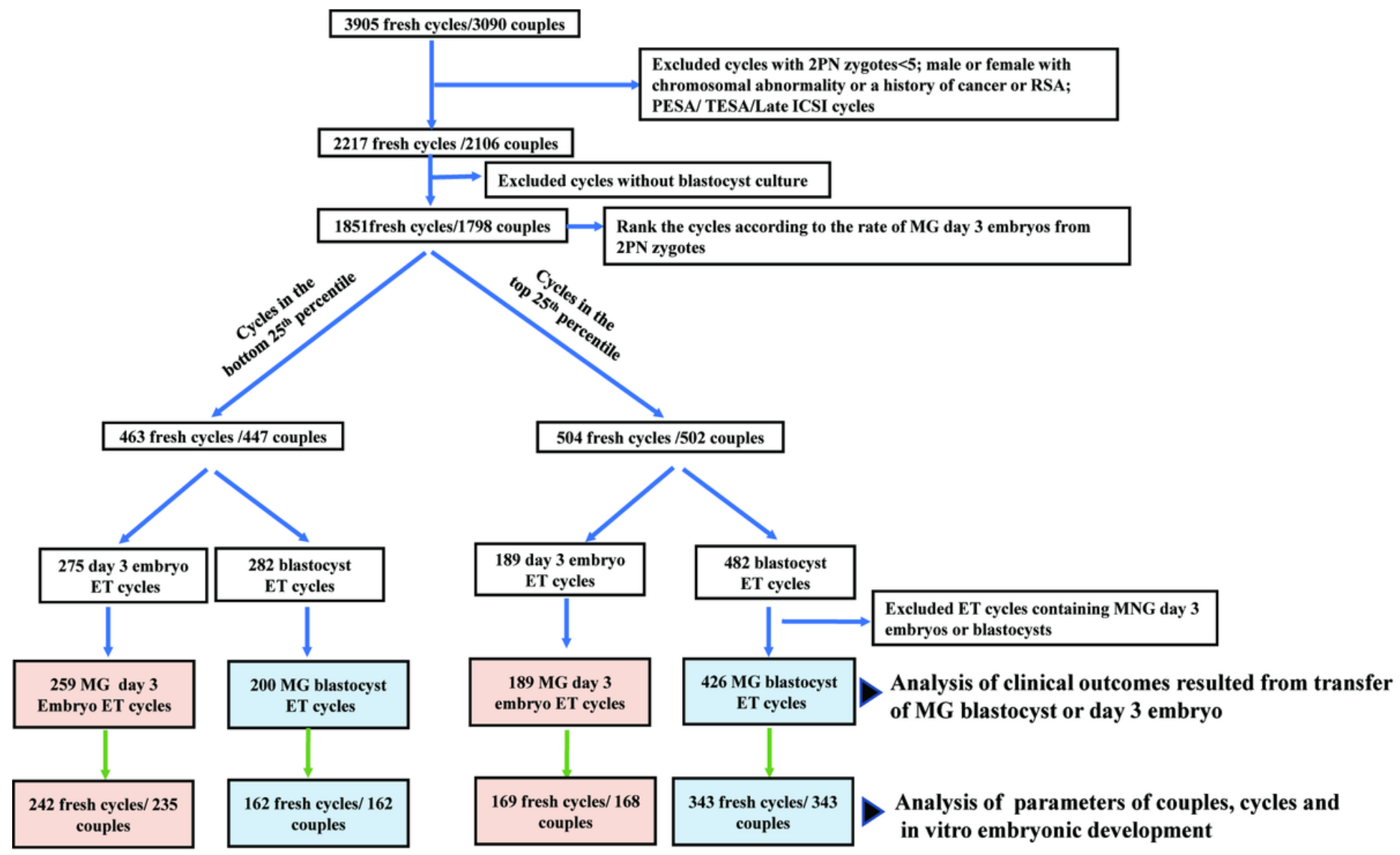

Figure 1

The flow chart of the present study. Following the exclusion of cycles with 2 PN zygotes $<5$ or without blastocyst culture, male or female with a history of cancer or chromosomal abnormality, or female having a diagnosis of recurrent spontaneous abortion, a total of 1851 fresh cycles was left for analysis. Cycles were ranked by the rate of morphologicallygoodday3 embryos. The cycles in the bottom 25 th percentile were defined as the $L$ group, while the cycles in the top 25th were defined as the $\mathrm{H}$ group. The data from 463 fresh cycles in the $L$ group and 504 fresh cycles in the $\mathrm{H}$ group contributed ET cycles was included.After the exclusion of ET cycles with MNG embryos, data from ET cycles in the $L$ group and $H$ groups were collected for analysis of clinical outcomes of transfer of the MG blastocysts and day 3 embryos. Then ET cycles related fresh cycles were collected to analyze the parameters of couples, cycles and in vitro embryonic development. 
$\mathrm{L}$

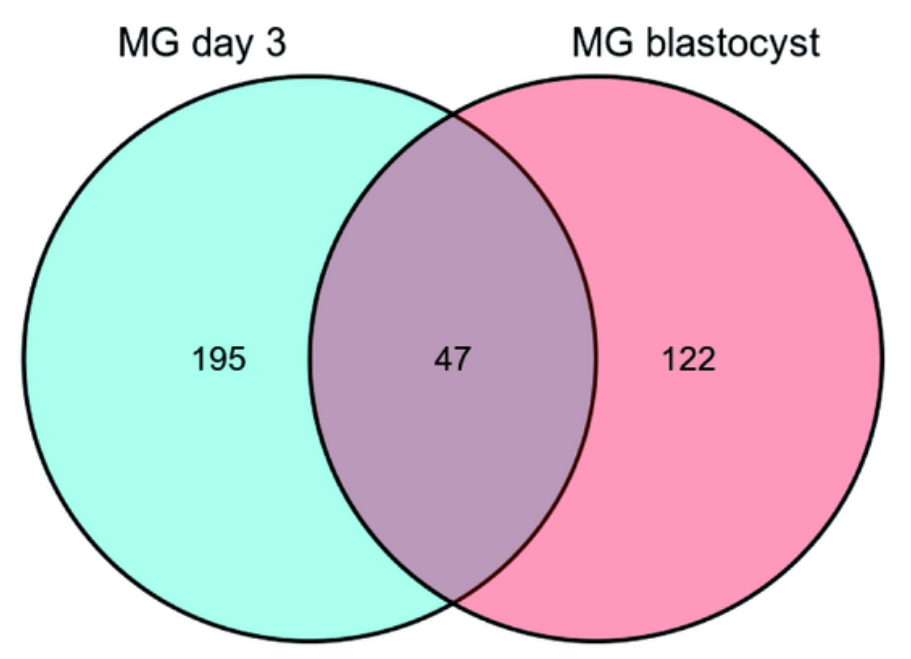

$\mathrm{H}$

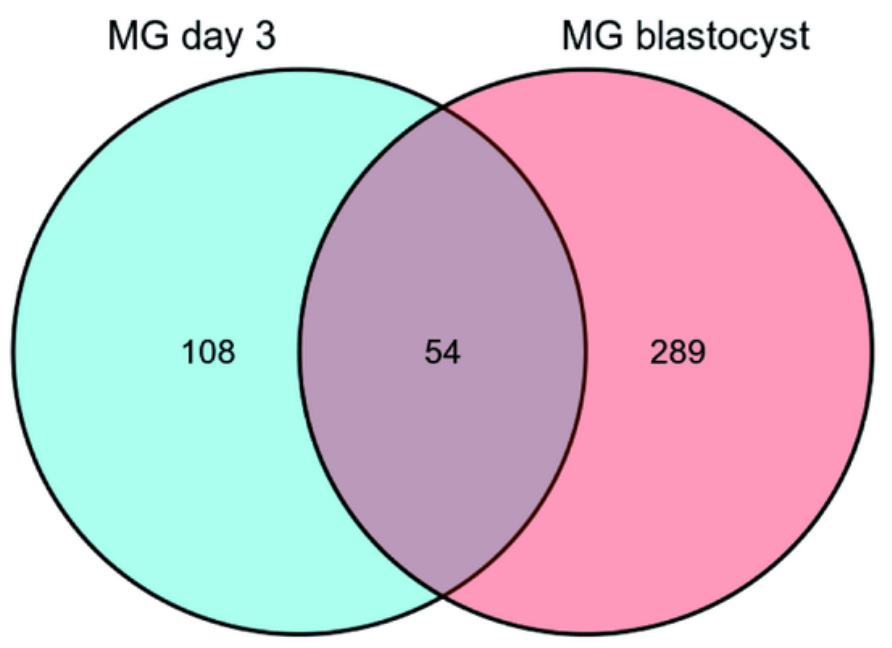

Figure 2

Fresh cycles with only MG day 3 embryo transfer and only MG blastocyst transfer, and fresh cycles with both MG day 3 embryo and blastocyst transfer in the L or $\mathrm{H}$ group. 195vs. 108 fresh cycles contributed only MG day 3 embryo transfer, 122 vs.289 fresh cycles contributed only MG blastocyst transfer and 47 vs.54 fresh cycles contributed both MG day 3 embryo and blastocyst transfer in the L vs. H group. 


\section{LRTD3 vs. HRTD3}

A
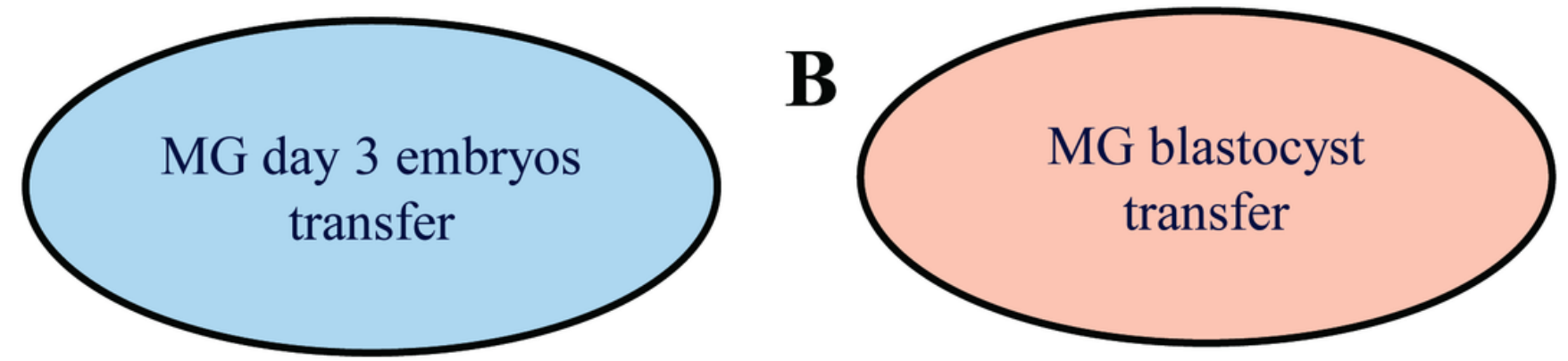

MG day 3 embryos

\section{MG day 3 embryos}

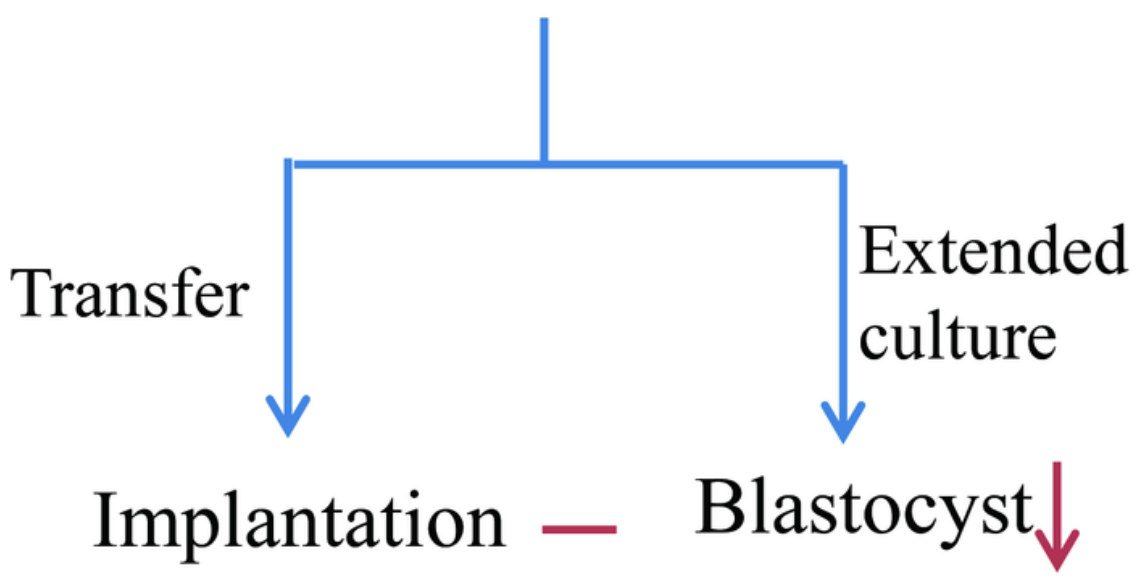

Extended

culture
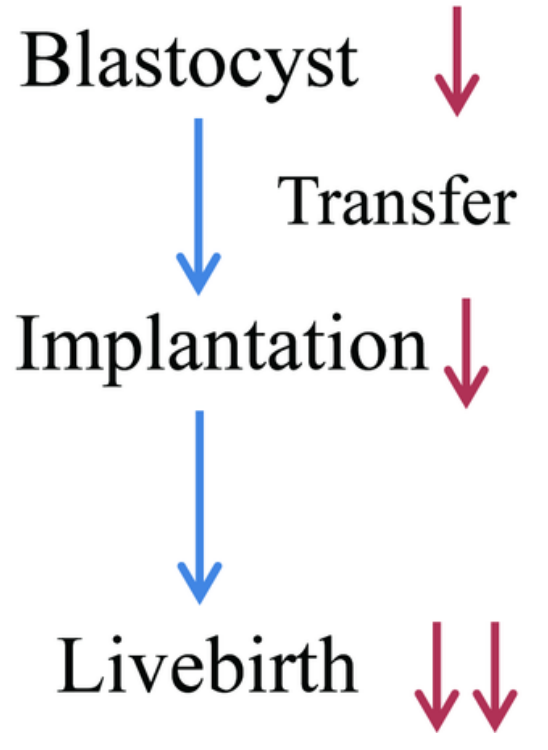

Figure 3

Summary findings. (A) For patients with transfer of MG day 3 embryos, patients with LRTD3 showed decreased rate of blastocyst formation and similar clinical outcomes of direct transfer of MG day3 embryos, compared to patients with HRTD3. (B) In patients with transfer of MG blastocysts, patients with LRTD3 showed decreased rate of blastocyst formation, as well as decreased rate of implantation and livebirth of transfer of blastocysts. 